\title{
NUMERICAL CALCULATION OF DEFORMATION OF THREE DIMENSIONAL SAMPLE IN TRIAXIAL APPARATUS UNDER EXTERNAL LOAD AND TEMPERATURE FIELD
}

\author{
MONIKA BARTLEWSKA-URBAN \\ Institute of Mining, Wrocław University of Technology, \\ Wybrzeże Wyspiańskiego 27, 50-370 Wrocław, Poland. E-mail: monika.bartlewska@pwr.wroc.pl

\section{TOMASZ STRZELECKI} \\ Institute of Geotechnics and Hydrotechnics, Wrocław University of Technology, \\ Wybrzeże Wyspiańskiego 27, 50-370 Wrocław, Poland. E-mail: tomasz.strzelecki@pwr.wroc.pl
}

\begin{abstract}
This study presents the results of calculations of the of thermo consolidation process of porous medium with the rheological Kelvin-Voigt skeleton, obtained numerically with the use of Flex.PDE. It is a continuation of the discussion on the phenomenon of thermal consolidation. A 3D problem considered boils down to solving the problem of the porous column filled with a liquid and treated by applying uniaxial compression load through a porous plate, allowing free flow of liquid from the center. To the sample affected by external lateral pressure. Numerical solution assumes compressing the sample at properly defined boundary conditions. The aim of this study was to describe the influence of external load and temperature gradient in the deformation tests for the case when the lateral surface is a good conductor of heat, and where the lateral surface of the sample does not conduct heat. The results obtained, in the context of further research, can also be used to determine the influence of other parameters of the state and model parameters on the process of thermo poroelasticity of the Biot model with rheological skeleton.
\end{abstract}

\section{INTRODUCTION}

The consolidation of the two-phase medium is the subject of numerous scientific publications. In these publications, each phase of the medium is assigned to the specific rheological model. The impact of temperature gradient on the process of deformation of porous medium can be called thermal consolidation process. The basic assumptions of the mechanics of soils and rocks were defined by Kisiel in [14]. Based on the mathematical model of creep of porous medium of Biot [8], [9] defined as the two-phase body, a number of studies were conducted. In a very general way, this model has been analyzed based on the theory of asymptotic homogenization method of periodic structures, where two-phase medium pores are filled with fluid that is just slightly compressible (Auriault [1], Bensoussan, Lions Papanicolau [10], Sanchez-Palencia [21]), or when it is filled by gas (Auriault, Strzelecki, Bauer and $\mathrm{He}$ [3]). The problem with the use of statistical methods was solved by Kröner [19], Rubinstein and Torquato [20]. 
Based on the fundamental postulate of the mechanics of porous medium that implies continuity of porous medium, it can be observed that, in fact, both models are proper for the mapping of the process of the center deformation, composed of discontinuous skeleton, (due to the different size and shape of the soil particles in the case of cohesive soils such as clay soils, clay, silts). Experimental studies indicate an important role of water layers - related to electric field forces which occur in this type in soils. The presence of water causes that within a significant temperature range, the particles of a skeleton interact with each other via this water. This issue has been dealt with by Bartlewska in her PhD thesis [5], as well as Bartlewska and Strzelecki in [6], [7], [26]. The problem of an impact of temperature field in the situation of adiabatic processes in the deformation process of two-phase medium composed of elastic skeleton and slightly compressible fluid was studied by Coussy. Based on the thermodynamics of irreversible processes, he developed a mathematical model of thermal consolidation in [12] and Kowalski and others in [16], [17]. The problem of adiabatic processes was also discussed by Strzelecki in [23], [24].

This work is a continuation of the numerical deliberation [6] about the impact of temperature on the deformation process. It includes a generalization of Biot's nonisothermal process equations including the rheological characteristics of the skeleton. The process of the displacement change as a function of time was analyzed according to different boundary conditions of numerically solved problem. In the first variant, an impact of external load and temperature gradient of the deformation process, the distribution of tensions and heat flow in the case of good heat conduction through the side wall of the sample was considered; the second variant involved isolating the sides of the sample by assuming a constant temperature of $20{ }^{\circ} \mathrm{C}$.

\section{THERMO COLSOLIDATION PROCESS EQUATIONS}

The mathematical equations presented below of the thermal consolidation process for the Biot body with rheological skeleton have been derived based on the fundamental laws of Newtonian mechanics for continuous center and thermodynamics of irreversible processes. The starting point are the initial assumptions of the theory of two-phase mediums composed from an elastic-viscous compressible skeleton and a viscous fluid filling the pores of this center. Assumptions are described in detail in [6].

Let $\Omega$ be the space defining element RVE (representative volume element) filled with a two-phase medium and limited by the surface $S$. Vector $\vec{n}$ is a unit vector normal to the surface $S$ facing the outside of the element $\Omega$. If $\vec{v}^{l}$ and $\vec{v}^{s}$ are respectively the vectors of the speed of liquid and skeleton, then $\vec{v}^{r}=\vec{v}^{l}-\vec{v}^{s}$ specifies a relative speed of the filtration flow of the liquid through the porous medium. If $\rho_{s}$ and $\rho_{l}$ stand for the specific density of the skeleton and the fluid, respectively, we can make a volumetric density of the skeleton $\rho_{1}=(1-f) \rho_{s}$ and of the fluid $\rho_{2}=f \rho_{l}$, 
where $\rho$ is the volumetric density of the two-phase medium equal in value to the total sum of $\rho_{1}+\rho_{2}$. The value of $\bar{\rho}$ is the density of the liquid flowing through the surface $S: \bar{\rho}=f_{s} \rho_{l}$.

The continuity equation of the two phases center is

$$
\int_{S} \rho v_{i}^{s} n_{i} d S+\int_{S} \rho v_{i}^{r} n_{i} d S+\int_{\Omega} \frac{\partial \rho}{\partial t} d \Omega=0 .
$$

Taking into consideration in the above equation the Gauss-Ostrogradski theorem, we can write down the above equation in the form of a local relationship

$$
\frac{D^{S} \rho}{D t}+\rho \dot{\varepsilon}=-\left[\bar{\rho} v_{i}^{r}\right]_{i}
$$

where $\frac{D^{S}}{D t}=\frac{\partial}{\partial t}+v_{i}^{s} \frac{\partial}{x_{i}}$ is the material derivative.

The equation of the continuity of the fluid flow through the skeleton of medium is given by

$$
\int_{S} \bar{\rho} v_{i}^{s} n_{i} d S+\int_{S} \bar{\rho} v_{i}^{r} n_{i} d S+\int_{\Omega} \frac{\partial \bar{\rho}}{\partial t} d \Omega=0
$$

Considering the Gauss-Ostrogradski theorem we can write down the above equation in the form of a local relationship

$$
\frac{D^{s} \bar{\rho}}{D t}+\bar{\rho}(\dot{\theta}-\dot{\varepsilon})=-v_{i}^{r}[\bar{\rho}]_{i} .
$$

The $\dot{\theta}$ and $\dot{\varepsilon}$ mean accordingly the rate of change of dilatation fluid and soil skeleton. The equations of movement of the solid phase medium are

$$
\int_{S} \sigma_{i j} n_{j} d S+\int_{\Omega} b v_{i}^{r} d \Omega+\int_{\Omega}(\rho-\bar{\rho}) X_{i} d \Omega=\int_{\Omega}\left(\rho_{11} \dot{v}_{i}^{s}+\rho_{12} \dot{v}_{i}^{l}\right) d \Omega
$$

where $b$ is the filtration coefficient of viscous resistance, and $\rho_{11}+\rho_{12}=\rho>0, \rho_{12}<0$. Local relationship which defines the equation of laminar motion of skeleton for quasistatic problems is reduced to the form

$$
\sigma_{i j}+X_{i}(\rho-\bar{\rho})=-b v_{i}^{r} .
$$

The equation of motion of the liquid phase in the case of laminar motion is expressed by the formula 


$$
\int_{S} \sigma n_{i} d S-\int_{\Omega} b v_{i}^{r} d \Omega+\int_{\Omega} X_{i} \rho d \Omega=\int_{\Omega}\left(\rho_{12} \dot{v}_{i}^{s}+\rho_{22} \dot{v}_{i}^{l}\right) d \Omega
$$

where $\rho_{12}+\rho_{22}=\rho_{2}>0$. Local relationship which defines the equation of laminar motion of fluid for the case of quasi-static issues is reduced to the form

$$
\sigma_{, i}+X_{i} \bar{\rho}=b v_{i}^{r}
$$

where $X_{i}=-\delta_{i 3} g$ is the gravitational acceleration in the right-hand frame of reference, $\sigma_{i j}$ the components of the stress tensor in the skeleton related to the total crosssectional area, $\sigma=-p * f$ the diffuse tension of a liquid filling the center of a porous medium.

Constitutive relationships for the Biot body with a rheological skeleton of KelvinVoigt for the adiabatic processes are presented

$$
\left\{\begin{array}{l}
\sigma_{i j}=2 N \varepsilon_{i j}+M \varepsilon \delta_{i j}+2 N T_{a} \dot{\varepsilon}_{i j}+\left(A T_{b}+N T_{a}\right) \dot{\varepsilon} \delta_{i j}+\frac{Q}{R} \sigma \delta_{i j}+P_{1}\left(T-T_{0}\right) \delta_{i j} \\
\sigma=Q \varepsilon+R Q+d\left(T-T_{0}\right)
\end{array}\right.
$$

where $N$ is the shear module of the skeleton, $A$ - module volume deformations of the skeleton, $Q$ - volumetric strain rate effect on the tension of the liquid in the shell or vice versa, the volumetric strain rate effect on the skeleton of stress in the liquid, $R$ - module of volume deformations of the liquid filling the pores of the Biot body. The parameter $M$ is expressed by:

$$
M=A-\frac{Q^{2}}{R} .
$$

Constant $d$ is given by

$$
d=-\left[3 Q r^{s}+r^{l} R\right]
$$

where $r^{s}$ and $r^{l}$ present accordingly the linear expansion of the skeleton and the volumetric expansion of the liquid.

Constant $P_{1}$ is calculated by the formula

$$
P_{1}=-\frac{T\left(3 K r^{s}+Q r^{l}\right)}{\lambda}
$$

where $\lambda$ is the heat transfer coefficient of soil.

$T_{a}$ and $T_{b}$ are the parameters of the skeleton expressed by the formulas

$$
T_{a}=\frac{\eta^{s}}{N} \text { and } T_{b}=\frac{\lambda^{s}}{A}
$$

$\eta^{s}, \lambda^{s}$ are the shear and volume viscosity of soil skeleton. 
The simultaneous equations of the linear theory of thermal consolidation in the movements of the skeleton and the function of stress in the liquid, the filtration flow equation and the heat conduction equation for the Biot body with a rheological Kelvin-Voigt skeleton consists of five differential equations

$$
\left\{\begin{array}{l}
N \Psi_{k} \nabla^{2} u_{i}+\left(A \Psi_{L}-\frac{Q^{2}}{R}+N \Psi_{k}\right) \varepsilon_{, i}+\frac{H}{R} \sigma_{, i}-\rho g \delta_{i 3}=-P_{1} T_{, i} \\
\frac{k R}{f_{o}^{2} \bar{\rho} g} \nabla^{2} \sigma=T_{0}\left[\dot{\sigma}-H \dot{\varepsilon}+P_{4} T\right] \\
\lambda \nabla^{2} T=T_{0}\left[P_{2} \dot{\varepsilon}-P_{3} \dot{\sigma}+P_{5} \dot{T}\right]
\end{array}\right.
$$

where $\Psi_{k}=1+T_{a} \frac{\partial}{\partial t}, \Psi_{L}=1+T_{b} \frac{\partial}{\partial t}$ are differential operators, $k$ is the coefficient of filtration of fluid through a porous medium, $g$ - gravitational acceleration, and coefficients $P_{2}, P_{3}, P_{4}$ and $P_{5}$ are given by the equations

$$
\begin{gathered}
P_{2}=3 r^{s}\left(K-\frac{H Q}{R}\right)-R r^{l}, \quad P_{3}=3 r^{s} \frac{Q}{R}+r^{l}, \quad P_{4}=R P_{3}, \\
P_{5}=\frac{\left(3 Q r^{s}+r^{l} R\right)^{2}}{R}+\frac{\left(\bar{\rho}_{s}+\bar{\rho}_{w}\right) c_{v}}{T},
\end{gathered}
$$

$c_{v}$ is the specific heat at constant volume. The above set of equations is a starting point for the issue resolved in the study.

\section{CONSTRUCTION OF NUMERICAL MODEL FOR THERMAL CONSOLIDATION OF ISSUES TRIAXIAL}

The process of thermal consolidation of porous media is described by the set of equations (10), on the basis of which a three-dimensional model of the center was created. Calculations were performed using the finite element using FlexPDE v. 6 Professional. Effective parameters of the model are constant and do not depend on the stress nor temperature. The effective parameters adopted for the calculations are shown in Tables 1 and 2.

For calculation purposes a sample in the shape of a cylinder was taken, with the radius of base $r=0.025 \mathrm{~m}$ and height $h=0.076 \mathrm{~m}$. The behavior of the samples was observed in the time range from $10^{-4}$ to $10^{10} \mathrm{~s}$ under the influence of the applied load 
$2 * 10^{6} \mathrm{~N} / \mathrm{m}^{2}$ to the upper part of the sample at the initial moment and the temperature gradient between the lower and upper surfaces of the sample was $80{ }^{\circ} \mathrm{C}$ in the time interval from $10^{5}$ to $10^{7} \mathrm{~s}$. For the rest of the time it was assumed that the temperature of the upper and lower surfaces of the sample is $20{ }^{\circ} \mathrm{C}$. For calculation of noninsulated wall side variant of the sample it is assumed that temperature of the wall side is $20{ }^{\circ} \mathrm{C}$. On the upper surface of the sample, horizontal components of the displacement skeleton's vector are assumed to be zero. The lower surface of the sample was defined as impervious to water and on top of their value in water pressure equal to the atmospheric pressure. A constant value of the pressure on the side walls of the sample was assumed to be $5 * 10^{5} \mathrm{~N} / \mathrm{m}^{2}$.

Table 1

Effective parameters of the Biot model with Kelvin-Viot skeleton

\begin{tabular}{|c|c|c|c|c|c|}
\hline$N$ & $A$ & $R$ & $Q$ & $\eta$ & $\lambda_{s}$ \\
\hline $\mathrm{N} / \mathrm{m}^{2}$ & $\mathrm{~N} / \mathrm{m}^{2}$ & $\mathrm{~N} / \mathrm{m}^{2}$ & $\mathrm{~N} / \mathrm{m}^{2}$ & $\mathrm{~Pa} * \mathrm{~s}$ & $\mathrm{~Pa} * \mathrm{~s}$ \\
\hline $2.5 * 10^{7}$ & $5 * 10^{7}$ & $1.5^{*} 10^{7}$ & $2.7 * 10^{7}$ & $2.2^{*} 10^{6}$ & $10^{7}$ \\
\hline
\end{tabular}

Table 2

Other effective parameters of soil

\begin{tabular}{|c|c|c|c|c|c|}
\hline $\mathrm{k}$ & $\mathrm{f}$ & $c_{v}$ & $\lambda$ & $r^{s}$ & $r^{l}$ \\
\hline $\mathrm{m} / \mathrm{s}$ & - & $\mathrm{J} /(\mathrm{kg} \mathrm{K})$ & $\mathrm{J} /(\mathrm{ms} \mathrm{K})$ & $1 / \mathrm{K}$ & $1 / \mathrm{K}$ \\
\hline $10^{-6}$ & 0.35 & $2 * 10^{3} \div 8^{*} 10^{3}$ & $0.5 \div 1.5$ & $5^{*} 10^{-5}$ & $10^{-7}$ \\
\hline
\end{tabular}

\section{RESULTS OF NUMERICAL CALCULATIONS}

Flex PDE system has a module automatically generating finite elements. The fixed network was used that took the split into 30 sections for each dimension, giving in result 50592 elements. The second variable element in the case of the transient processes, like this one, is a time step $d t$. The program starts running with the time step starting $d t=10^{-5} \mathrm{~s}$, and it ends with $d t=2.96^{*} 10^{9} \mathrm{~s}$. In Figs. 1 and 2, the distribution of the finite elements at the starting stage of the calculation is presented to conduct the comparative analysis; the following figures present the graphs: displacements, distribution of temperature, heat flow and the $z$-axis - directed tension of the external load accordingly for the two variants analyzed. 


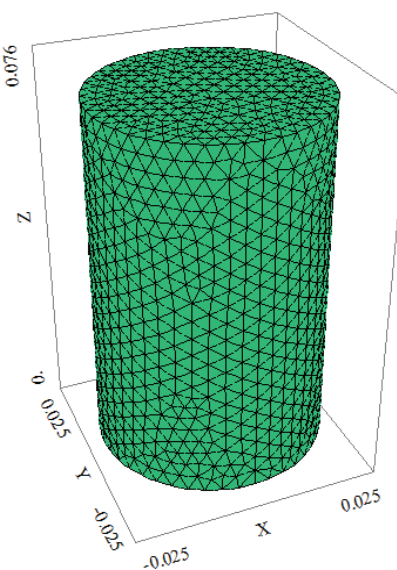

Fig. 1. Finite element mesh generated by the software

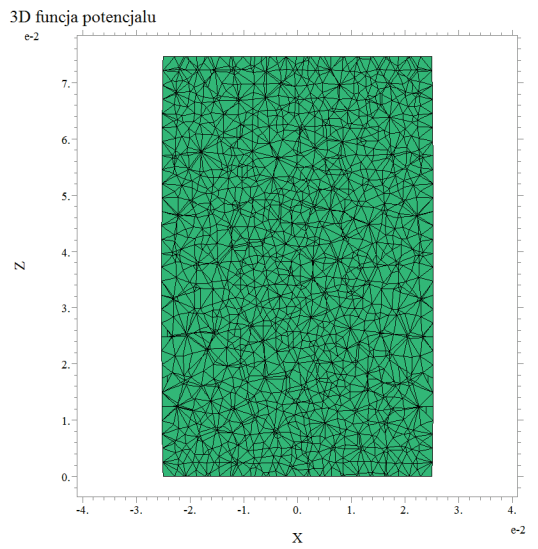

przep01adap: Cycle $=28$ Time $=0.1000 \mathrm{dt}=0.0200 \mathrm{p} 2$ Nodes $=74437$ Cells $=50592 \mathrm{RMS}$ Err $=0.0319$

Fig. 2. Calculational mesh

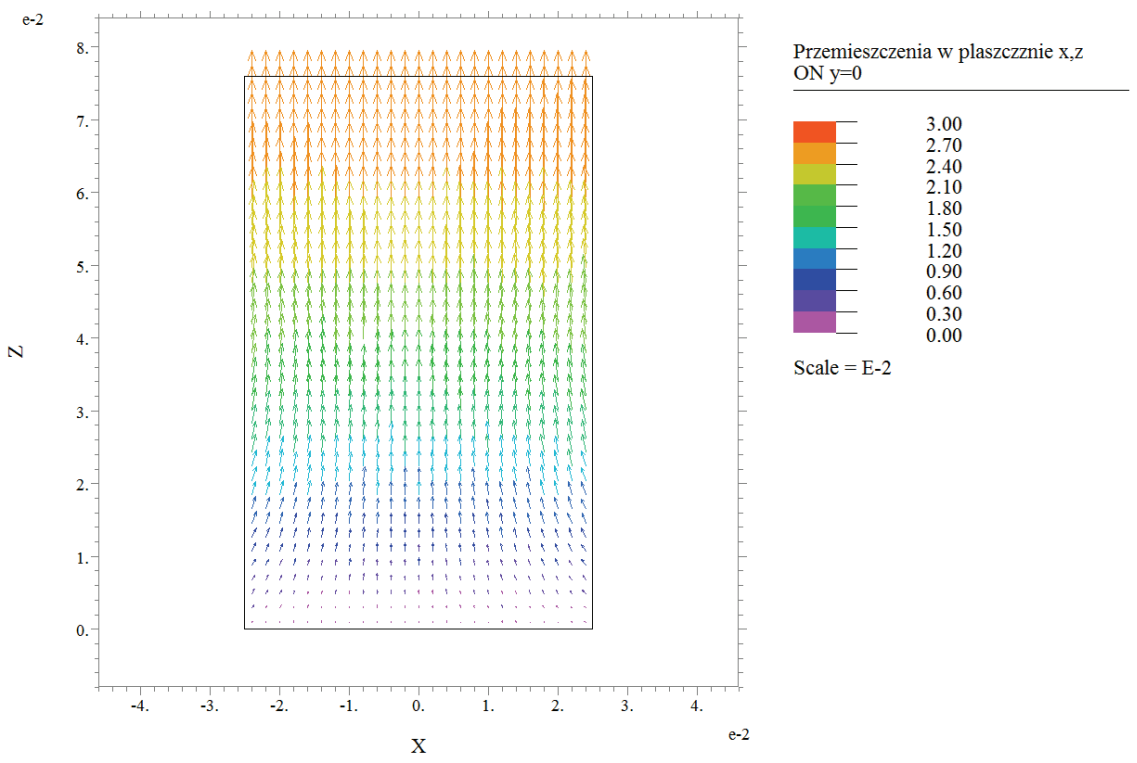

Fig. 3. Displacement vectors in vertical plane at $t=1 * 10^{6} \mathrm{~s}$ for wall side non-insulated sample

In the first variant considered, the lateral surface of the sample does not conduct the heat and in the second one, it conducts the heat. All the graphs present the same moment of the experiment $t=1 * 10^{6} \mathrm{~s}$, taking ito consideration the time interval required for the sample to be heated (from $10^{5} \mathrm{~s}$ to $10^{7}$ ), that is, the moment when the soil is exposed in this experiment to the maximum temperature of $80{ }^{\circ} \mathrm{C}$. In Figures 3 and 4 , the vectors of the displacements are presented for two variants of the heat conduct 
through the lateral surface of the sample for the same time of the simulation: $t=$ $1 * 10^{6} \mathrm{~s}$ (heating time). The values of the displacements for the side wall non-insulated sample are bigger than in the case where the walls are insulated. Both figures differ also in the direction of the displacement vectors at the lower part of the sample that stays heated. In this case, we can observe that for the non-insulated sample the biggest displacements in the horizontal direction are in places of the highest temperature.

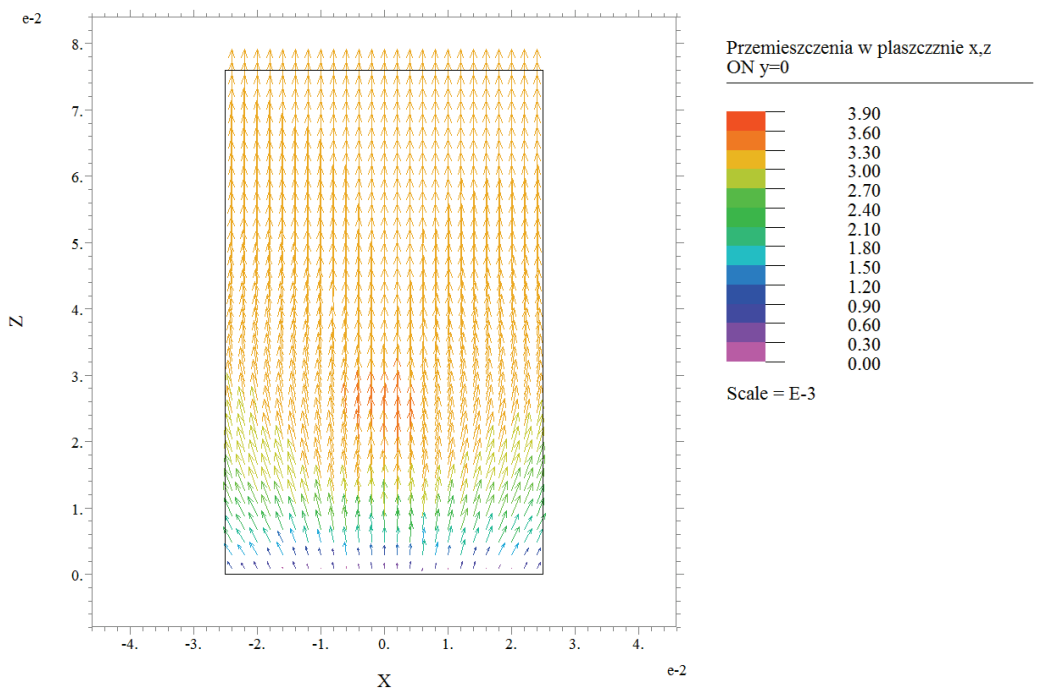

Fig. 4. Displacement vectors in vertical plane at $t=1 * 10^{6} \mathrm{~s}$ for side insulated sample

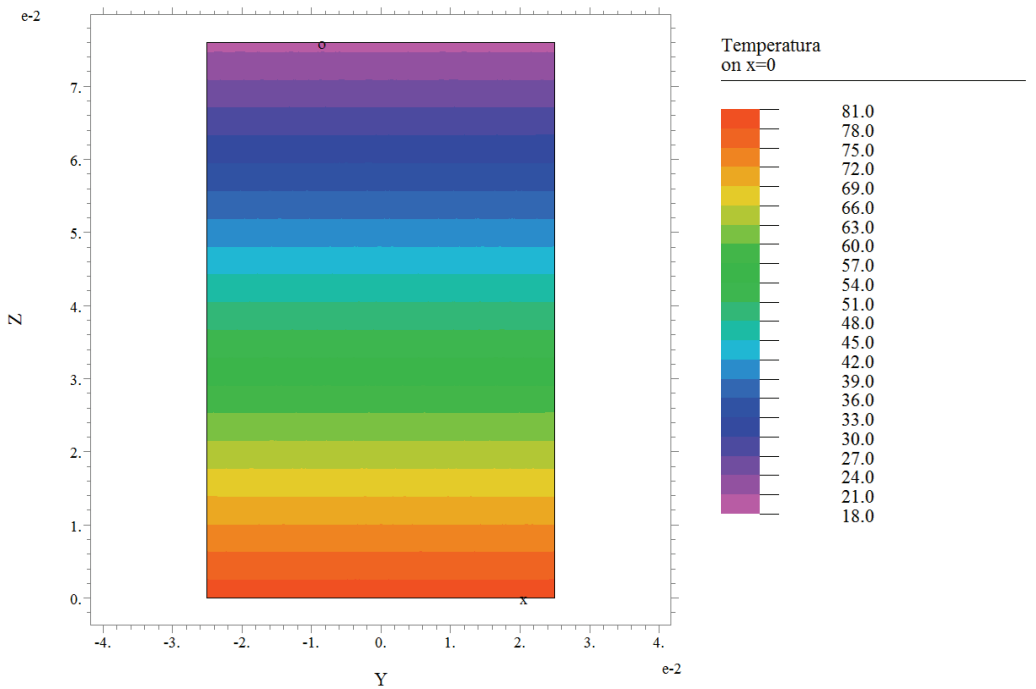

Fig. 5. Contours of temperature at $t=1 * 10^{6} \mathrm{~s}$ for side non-insulated sample 


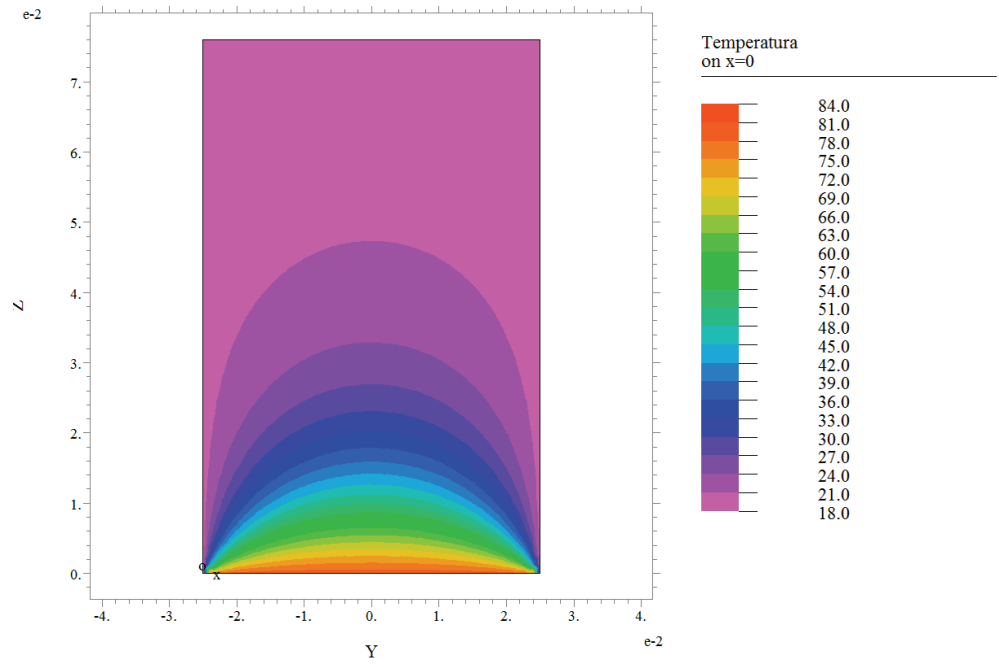

Fig. 6. Contours of temperature at $t=1 * 10^{6} \mathrm{~s}$ for side insulated sample

Both temperature distribution (Fig. 5 and Fig. 6) and heat flow field are significantly different for each variant. In the side insulated sample we can observe a regular temperature distribution as well as homogeneous vertical flow from the warmer surface. For the sample non-insulted laterally we can observe the outflow of the heat through non-insulted surface which is a direct reason of the lower temperature (close to the state before heating) in most part of the sample (Fig. 6).

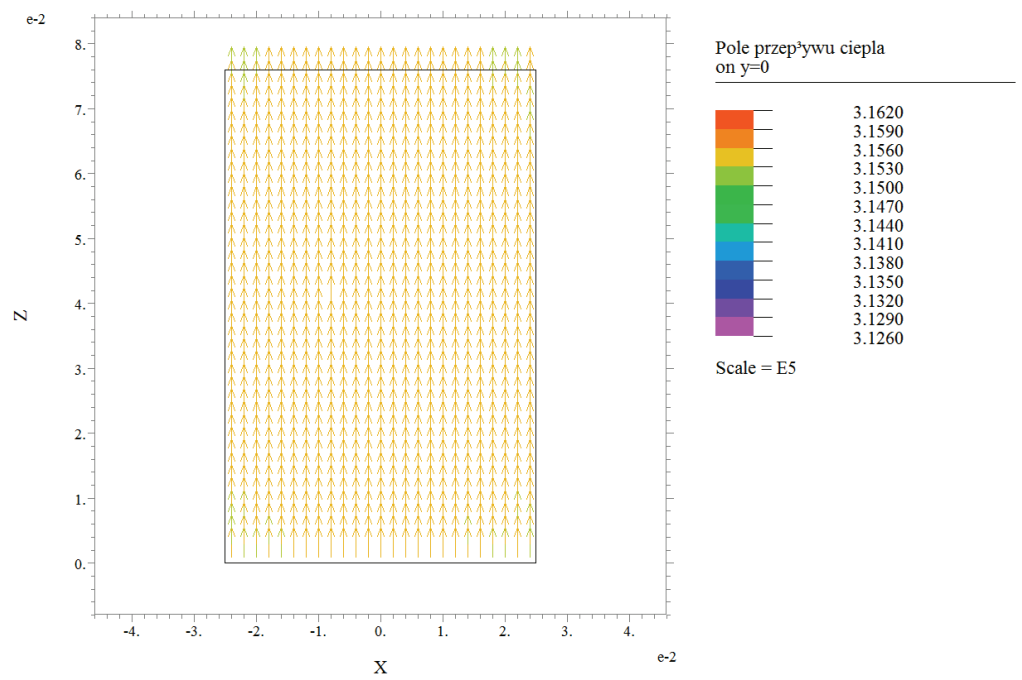

Fig. 7. Temperature flow field at $t=1 * 10^{6} \mathrm{~s}$ for side insulated sample 


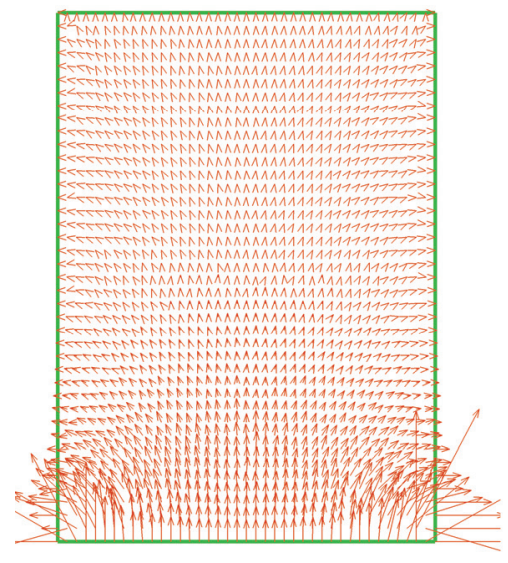

Fig. 8. Temperature flow field at $t=1 * 10^{6} \mathrm{~s}$ for side non-insulated sample

Stress distribution in the vertical direction in the case of the sample with heat impermeable walls (Fig. 9) tends to regular distribution, the biggest tensions in the time $t=1 * 10^{6} \mathrm{~s}$ are on the top side of the sample exactly in the place of the external load and at the bottom where the sample is heated. The stress decrease towards the sample's center after finishing the heating process achieves its minimal value at the sample's bottom. Whereas in the case of the sample with heat (Fig. 10) conducting walls, the vertical tensions take the biggest values at the sample's bottom at its central point radially decreasing towards the side wall to finally achieve its minimum in the corners at the bottom. In the remaining parts of the samples they are constant. We can thus observe significant differences in the distribution of vertical tensions for both variants.

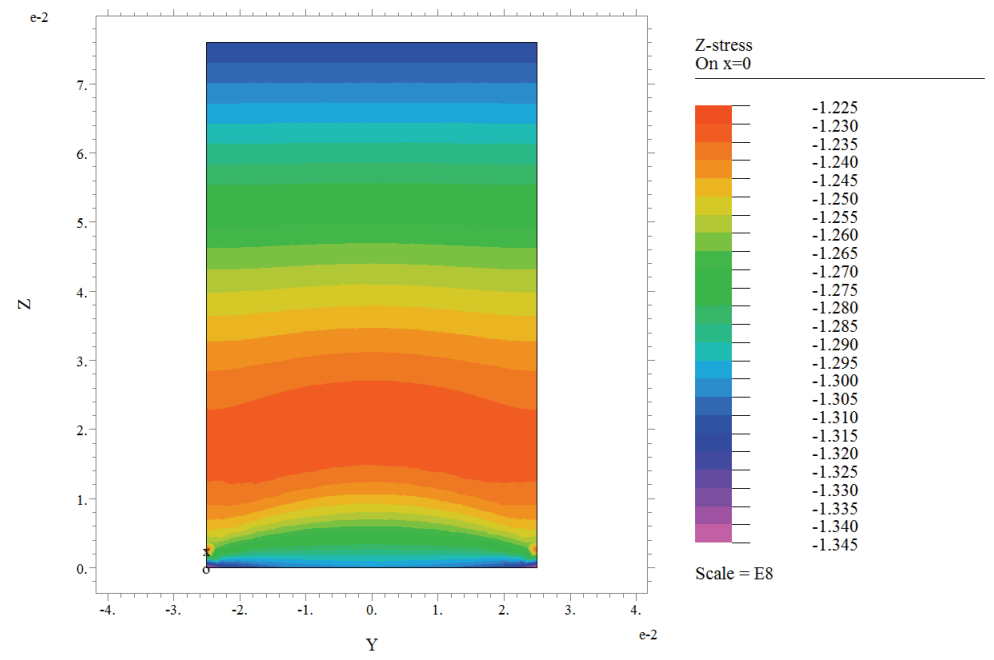

Fig. 9. Z-stress in vertical plane at $t=1 * 10^{6} \mathrm{~s}$ for side insulated sample 


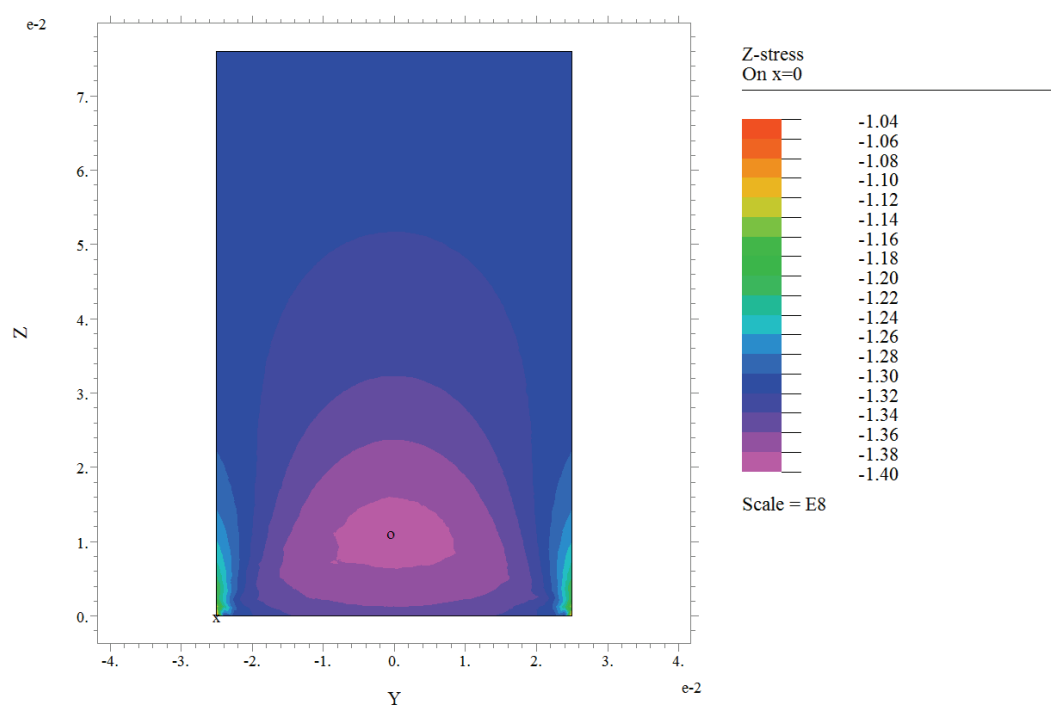

Fig. 10. Vertical stress displacement for $t=1 * 10^{6} \mathrm{~s}$ (for the sample with a lateral surface is a good heat conductor)

\section{SUMMARY AND CONCLUSIONS}

In this paper, the authors proposed to include in the soil consolidation process, the impact of temperature in the process of evolution of the porous-medium including changes of the shear and volume viscosity of the skeleton, as well as viscosity on the flowing fluid influenced by the field of temperature. A mathematical model of thermal consolidation presented in the study, which includes the rheological features of the Kelvin-Voigt skeleton, is a more detailed approximation of real processes, as its important aspect is the lack of immediate displacements. The numerical solution has assumed compressing the sample under the properly defined boundary conditions. The process of the change in displacements as a function of time, temperature and tension was analyzed in situation where the lateral surface of the sample is a good heat conductor and in the situation when it is not. The results of the numerical simulation in both cases differed significantly. Bigger vertical displacements were observed for the sample with a well conducting lateral surface. The temperature distribution and the direction on the heat vectors in the isolated sample had a regular character - the highest where the load worked or where the sample was heated. Whereas in the second sample the heat drained away through the lateral surfaces so the fundamentally higher, during heating, temperature was observed only at the bottom of the sample - the rest of the material examined had a low starting temperature, practically not heating at all. The graphs of displacements, tension distribution and field of the heat flow, were ob- 
tained directly from the FlexPDE v.6 program and are compatible with the experiment assumptions as well as with the intuition and indicate the correctness of the model. The model can be used to help resolving problems that are important from the engineering perspective, especially the topics considering the tension-distortion states in rocks at greater depths that require comprehensive analysis of the geothermal processes and their impact on the rocks in the field of a significant temperature gradient. This may be important with regard to the plans of building generators of coal gasification.

\section{REFERENCES}

[1] Auriaullt J.L., Dynamic behaviour of porous media, Transport Processes in Porous Media, Kluver Academic Publishers, 1991, 471-519.

[2] Auriault J.L., Sanchez-Palencia E., Etude de comportement macroscopique d'un milieu poreux sature deformable, Journal de Mecanique, 16(4), 1977b, 575-603.

[3] Auriault J.L, Strzelecki T., Bauer J., He S., Porous deformable media by a very compressible Fluid, Eur. J. Mech. a/Solid, 9, 4, 1990, 373-392.

[4] BartLeWSKa-Urban M., STRZEleCKi T., One-dimensional consolidation of the porous medium with the rheological Kelvin-Voigt skeleton, Studia Geotechnica et Mechanica, Vol. XXX, No. 1/2, 2008, $115-122$.

[5] BARTLEWSKa M., The doctoral dissertation on the theme: Determination of effective parameters of rheological models of cohesive soils, Politechnika Wrocławska, Faculty of Geoengineering, Mining and Geology, Wrocław, 2009, (in Polish).

[6] BARTLEWSKa M, STRZELECKI T., Equations of Biots consolidation with Kelvin-Voight rheological frame, Studia Geotechnica et Mechanica, Vol. XXXI, No. 2, 2009, 3-15.

[7] BartlewsKa M., StrZeleCKi T., One-dimensional consolidation of the porous medium with the Rheological Kelvin-Voight skeleton, Studia Geotechnica et Mechanica, Vol. XXX, No. 1-2. 2008.

[8] Віот M.A., General theory of three-dimensional consolidation, J. Appl. Phys., No 12, 1941, p. 155.

[9] Biot M.A., General Solutions of the Equations of Elasticity and Consolidation of a Porous Material, J. Appl. Mech., 1956, 23.

[10] Bensoussan A., Lions J.L., Papanicolau G., Asymptotic analysis for periodic structures, North Holland Publishing Company, Amsterdam, 1978,

[11] Coussy O., Mechanics of Porous Continua, John Wiley \& Sons, 1995.

[12] Coussy O., Mechanics and physics of porous solids, John Wiley \& Sons, 2011.

[13] Kisiel I., Derski W., IzBicki R.J., Mróz Z., Soils and rocks mechanics, series Mechanika Techniczna, Vol. VII, PWN, Warszawa, 1982, (in Polish).

[14] Kisiel I., Rheological state equation of quasilinear medium, Polish Academy of Sciences - Wrocław Branch, Wrocław, 1980, (in Polish).

[15] Kowalski S.J., Musielak G., RYbicki A., Drying Processes in Context of the Theory of Fluid Saturated Porous Materials, J. Theoretical and Applied Mechanics, 36, 3, 1998.

[16] Kowalski S.J., MusielaK G., Drying Processes in Aspect of the Theory of the Fluid Saturated Porous Materials, Proceedings of the Fifth International Conference on Composities Engineering, ICCE/5, ed. D. Hui, 1998, 487-488.

[17] Kowalski S.J., MusielaK G., Rybicki A., Theory of Drying Processes as an Aspect of Poromechanics, "Poromechanics - A Tribute to Maurice A. Biot", J.F. Thimus et al. (ed.), A.A. Balkema, Rotterdam-Brookfield, 1998, 433-437. 
[18] KRÖNER E., Effective elastic moduli of periodic and random media: a unification, Mechanics Research Communication, 7(5), 1980, 323-327.

[19] ReINER M., Deformation, strain and flow, H.K. Lewis, London, 1960.

[20] Rubinstein J., Torquato S., Flow and random porous media: mathematical formulation, variational principles and rigorous bounds, J. Fluid Mech., 206, 1989, 25-46.

[21] Sanchez-Palencia E., Non homogeneous Media and Vibration Theory, Lecture Notes in Physics, 127, Springer-Verlag, Berlin, 1980.

[22] STRZELECKI T., Loi de comportement dans la theorie de la consolidation electrohydrodynamique, Stud. Geotech., Vol. 1, No. 3/4, 1979.

[23] STRZELECKI T., Model of thermo-consolidation processes of clay soil taking into account electrokinetic processes, Współczesne problemy naukowo badawcze budownictwa lądowego i wodnego, Oficyna Wydawnicza Politechniki Wrocławskiej, 2007 (in Polish).

[24] Strzelecki T., Kostecki S., ŻAK S., Modelling of flow through porous media, Dolnośląskie Wydawnictwo Edukacyjne, 2008, (in Polish).

[25] SzefER G., Non-linear Problems of consolidations theory, Mat. III Kolokwium Polsko-Francuskiego, 22-24 April 1980.

[26] StrZelecki T., BartlewsKa-Urban M., Numerical calculations of the consolidation of flotation waste landfill "Żelazny Most" based on Biot's model with the Kelvin-Voight rheological skeleton, Archives of Civil Engineering, Vol. 57, Iss. 2, 2011, 199-213.

[27] Flex PDE v.6 : www.pdesolutions.com. 